\title{
CANTOR ON INFINITESIMALS HISTORICAL AND MODERN PERSPECTIVE
}

\begin{abstract}
In his 1887's Mitteilungen zur Lehre von Transfiniten, Cantor seeks to prove inconsistency of infinitesimals. We provide a detailed analysis of his argument from both historical and mathematical perspective. We show that while his historical analysis are questionable, the mathematical part of the argument is false.
\end{abstract}

Keywords: Infinitesimals, infinite numbers, real numbers, hyperreals, ordinal numbers, Conway numbers.

\section{Introduction}

It is well-known that Cantor praised Bolzano for developing the arithmetic of proper-infinite numbers. The famous quotation reads:

"Bolzano is perhaps the only one for whom the proper-infinite numbers are legitimate (at any rate, he speaks about them a great deal); but I absolutely do not agree with the manner in which he handles them without being able to give a correct definition, and I regard, for example, $\S \S 29-33$ of that book [Paradoxien des Unendlichen] as unsupported and erroneous. The author lacks two things necessary for a genuine grasp of the concept of determinate-infinite number: both the general concept of power and the precise concept of Anzahl". ${ }^{1}$

*The first author is supported by the National Science Centre, Poland grant 2018/31/B/HS1/03896.

${ }^{1}[15$, p. 181$]$ translated by W. Ewald [31, p. 895, note in square brackets added]. 
Interestingly, the specified paragraphs of Paradoxien develop calculus in a way that appeals to Euler's 1748 Introductio in Analysin Infinitorum, i.e., calculus that employs infinitely small and infinitely large numbers along with the relation is infinitely close. Euler's masterpiece was written in Latin, then it was translated into French by J. B. Labey in 1796, and into German by J. A. C. Michelsen in 1788-1791, another German translation by $\mathrm{H}$. Maser was released in 1885. It was arguably one of the most important 18 th century mathematical treaties. But there are only two significant references to [29] in Cantor's Gesammelte Abhandlungen: [12] and [13]. They deal with a specific issue raised by Euler in the section $\S 328$ called De partitione numerorum. Any other references to Euler are also of minor importance. Furthermore, in Cantor's Briefe [45] the name Euler occurs twice, and, again, in very short remarks. Quite strange.

Cantor has never addressed Euler's technique of infinite numbers as employed in determining infinite sums and products. Whether knowingly or unknowingly, or just by correcting supposed errors, in [12] and [13] he interprets Euler's infinite operations within the framework of standard analysis, albeit limits do not occur in [29]. In [16] he discusses infinitesimals through distinction actual vs. potential infinity. ${ }^{2}$ Due to the theory of limits, developed, among others, by Cauchy - Cantor argues - the mistake of ascribing them actual infinity had been fixed. The letter to Mittag-Leffler dated March 3, 1883 contains a hint that Euler's and Bolzano's infinite sums are inconsistent. ${ }^{3}$ Notes such as these as well as concerning infinitesimals are scattered all throughout Cantor's papers, however he has never developed them into a thorough criticism. Instead, they attest Cantor's aversion to infinitesimals - an aversion based on prejudices rather than concrete arguments.

As to Euler, in [30], the number line is explicitly revealed as consisting of infinitesimals, infinitely large numbers, and assignable quantities, i.e., numbers representing line segments, while infinite numbers are viewed as inverses of infinitesimals. In [29], infinitesimals and infinitely large numbers are employed to expand $\sin x$ and $\cos x$ functions into series, and then to derive the famous formula $e^{i x}=\cos x+i \sin x$, to mention the most spectacular achievements. Thus, Euler's infinite numbers provide a ma-

\footnotetext{
${ }^{2}$ See [16, p. 410].

${ }^{3}$ See [45, p. 117-118]. Another reference to Euler occurs in the letter to Lipschitz dated October 18, 1885 [45, p. 247]; it regards an arithmetic problem.
} 
chinery which enable crucial mathematical results. And yet, there are no references to this technique neither in Cantor's papers, nor in his letters. Why then, instead of referring to the mathematical treatise that explicitly develops the analysis of infinity, does Cantor prefer to discuss ancient Greek and medieval philosophers? Moreover, whereas his theory of infinity is formal, his philosophical considerations explore distinctions that he could never formalize, namely actual infinity vs. potential infinity. Instead, Euler's approach to infinity builds on the easily formalized opposition of finite vs. non-finite that turned out to be equivalent to the Archimedean vs. non-Archimedean opposition.

We believe that the following quotation is crucial when it comes to understanding Cantor's perspective:

"The fact of actual infinite numbers is thus so little ground for the existence of actual infinitely small magnitudes that, on the contrary, the impossibility of the latter can be proven with the former". ${ }^{4}$

In fact, there are many similar declarations scattered throughout Cantor's papers, showing that he considered infinitesimals as the most serious rival of his theory of infinite numbers.

Cantor's position is more understandable when we realize that he was absolutely certain about his characterization of infinity being the only possible characterization. Similarly, he was absolutely certain there was only one possible domain to develop the calculus. ${ }^{5}$ This no-alternative philosophy, whether applied to infinity or to a domain of calculus, motivated Cantor's struggle with infinitesimals.

In his 1887 paper Mitteilungen zur Lehre von Transfiniten, Cantor goes beyond declarations and seeks to prove that infinitesimals are inconsistent. The general idea of his argument is this: Let $\zeta$ be a positive infinitesimal, which, for him, means it fulfills the condition $(\forall n \in \mathbb{N})\left(\zeta<\frac{1}{n}\right)$, then for any infinite ordinal number $\nu$ the product $\zeta \cdot \nu$ is smaller than any finite magnitude, in symbols

$$
(\forall n \in \mathbb{N})\left(\zeta<\frac{1}{n}\right) \Rightarrow(\forall \nu \in O r d)(\forall n \in \mathbb{N})\left(\zeta \cdot \nu<\frac{1}{n}\right),
$$

where Ord stands for the class of ordinal numbers. In other words, provided $\zeta$ is infinitesimal, every product $\zeta \cdot \nu$ is also infinitesimal. Hereinafter, we will also refer to the more suggestive and equivalent version of (1), namely

\footnotetext{
4 [16, p. 408], translated by P. Enrlich [27, p. 42].

${ }^{5}$ See [22, pp. 233-236].
} 


$$
(\forall n \in \mathbb{N})\left(\zeta<\frac{1}{n}\right) \Rightarrow(\forall \nu \in O r d)(\zeta \cdot \nu<1) .
$$

Cantor's argument looks like a reductio ad absurdum proof, while the supposed contradiction is to consist of the following statements: $\zeta$ is a linear magnitude, $\zeta$ cannot be made finite through any actual infinite multiplication. Arguably, to get a real contradiction, Cantor's argument requires interpretation. One has to decide (a) what is the meaning of the term linear magnitude, (b) what does the product $\zeta \cdot \nu$ mean.

In this paper, we will provide a detailed analysis of Cantor's argument and will argue that linear magnitude means real numbers. We will also present some modern interpretations of the product $\zeta \cdot \nu$ that do not entail the conclusion $(\forall \nu \in O r d)(\zeta \cdot \nu<1)$.

\section{Linear magnitude and Archimedean property}

Provided that Cantor's linear magnitude means positive real numbers, his proof of the inconsistency of infinitesimals aims to show that the concept of linear magnitude implies the Archimedean property.

Indeed, Cantor's definition of infinitesimals is the same as the one provided in Bolzano's Paradoxien, $\S \S 10,16$; it is, in fact, the same definition as the modern one. Cantor, thus, sought to show that no infinitesimal is a real number. From the modern, axiomatic perspective, it is an obvious observation, as the completeness of the field of real numbers implies the Archimedean property, and the Archimedean property excludes infinitesimals. In fact, Cantor's reasoning in its full version involves the Archimedean property and can be paraphrased as follows: Linear magnitude has the Archimedean property, while infinitesimals and the Archimedean property are mutually exclusive.

However, the argument is not that simple, as Cantor adopts a specific interpretation of what we nowadays consider to be the Archimedean axiom, AA in short. Namely, he allows multiplications by any ordinal rather than any natural number. More importantly, his characteristic of real numbers differs from our modern one. In the 1872's Über die Ausdehnung eines Satzes der Theorie der trigonometrischen Reihen, he identified the completeness (continuity) of real numbers with a condition currently called Cauchy completeness, CC in short. While nowadays we know that AA does not follow from CC, this was not the case at the turn of the 19th and 20 th century. 
In 1887, neither Cantor nor Dedekind were quite sure whether their versions of continuity of real numbers were equivalent. It was partly because there was no obvious framework that would enable to establish or dismiss the equivalence of Cantor's and Dedekind's versions. Adopting a modern perspective, we can say that in [14] Cantor sought to characterize continuum as an ordered field $(\mathbb{R},+, \cdot, 0,1,<)$, in $[15]$ as a subset of the metric space $\mathbb{R}^{n}$, and in [17] as a totally ordered set $(\mathbb{R},<)$. The Dedekind cut principle does not apply to the subsets of metric space; in the context of totally ordered sets, it does not provide unique characteristics (i.e. up to isomorphism); in the framework of an ordered field, the cut principle implies CC, yet not vice versa. ${ }^{6}$ As all these facts were not clear at the time, it is no wonder Cantor objected whether the Dedekind cut principle really reveals the "essence of continuity". ${ }^{7}$ Basically - in our interpretation - it can-not be applied in every context he considered continuum.

In modern mathematics, the concept of an ordered field provides such a framework, yet the concept itself was introduced only in Hilbert's 1899 Grundlagen der Geometrie. The first widely-known proof that completeness of real numbers implies the Archimedean axiom was given in 1901 by Otto Hölder; to this end, he applied the Dedekind cut version of completeness, and the result was established for an ordered group. In 1900's Über den Zahlbegriff, Hilbert presents the continuity of real numbers in the form of a conjunction: AA plus Axiom of Completeness; the second condition could be paraphrased as follows: Real numbers are the biggest Archimedean field. In 1932's Anschauliche Geometrie, Hilbert characterized the continuity of real numbers as a conjunction of AA plus the condition, which he named Cantor's axiom, namely: If $\left(A_{n}\right)$ is a descending sequence of closed line segments, then $\bigcap_{n=1}^{\infty} A_{n} \neq \emptyset$.

In fact, we can not determine who was the first to show that $\mathrm{CC}$ does not imply AA. [19] proves that the field of Laurent series over real numbers is a non-Archimedean, Cauchy-complete field. [46] shows that Levi-Civita fields are non-Archimedean and Cauchy-complete. We can show that the field of hyperreals is yet another example of a non-Archimedean, Cauchycomplete field. Still, these are relatively recent results.

In what follows, next to Cantor's construction of real numbers and its accompanied characteristics of continuity, we will also discuss Cantor's

\footnotetext{
${ }^{6}$ We develop these claims in section 7 .

${ }^{7}$ The very phrase occurs in [23].
} 
topological characterization of linear magnitude given in [15], as well as his characterization of real numbers in terms of total order given in [17]. None of these characteristics implies the Archimedean property. Nevertheless, suggestions that the line of real numbers $(\mathbb{R},<)$ has the Archimedean property permeated Cantor's milieu.

Indeed, there were some earlier attempts to prove the Archimedean property in the 19th century. The first one we know of was made by Bolzano in his Reine Zahlenlehre; yet the paper was published posthumously in $1962 .{ }^{8}$ In 1817's Rein analytischer Beweis, he applied the seemingly self-evident condition $\lim _{n \rightarrow \infty} \frac{1}{n}=0$; nowadays we know that it is but another version of the Archimedean axiom. In 1885's Vorlesungen über allgemeine Arithmetik, Stolz sought to show that the Archimedean property follows from the Dedekind cut principle; his proof, however, proceeded in a geometrical framework. In the 1890's Teoria delle grandezze, Bottazzi proved that in the abelian group the Dedekind cut principle implies the Archimedean property. ${ }^{9}$

Whether correct or not, they were attempts to derive the Archimedean property from the Dedekind cut principle. ${ }^{10}$ Since Cantor could not decide whether his account of continuum differs from that proposed by Dedekind, it is no wonder he was seeking his own proof of the Archimedean property. Moreover, he knew that in some contexts, e.g. in a theory of totally ordered sets, the Dedekind cut principle was insufficient to characterize real numbers up to isomorphism. This could be the reason behind his search for a genuine proof.

The rest of paper is as follows: in section 3 we present basic mathematical facts concerning infinitesimals, the Archimedean axiom, continuity and related issues. Then, in subsequent sections, we provide a detailed analysis of chapter VI of [16] which includes Cantor's inconsistency proof of infinitesimals.

\footnotetext{
${ }^{8}$ See $[7, \S 69]$ and $[8, \S 74]$. There is, however, an alternative interpretation of these paragraphs to the effect that instead of proving the Archimedean property of real numbers, Bolzano sought to show that his measurable numbers had the Archimedean property, and to this end he assumed that real numbers had the Archimedean property. Then, Bolzano's measurable numbers are viewed as assignable hyperreal numbers.

${ }^{9}$ See $[27$, p. 80].

10 [1] identifies flaws in Stolz's 1885 proof. In fact, [48] provides a corrected version of his 1885 proof. Still, the 1902 proof is incomplete.
} 


\section{Basic Facts}

A commutative field $(\mathbb{F},+, \cdot, 0,1)$ together with a total order $<$ is an ordered field when the sums and products are compatible with the order, that is

$$
x<y \Rightarrow x+z<y+z, \quad x<y, 0<z \Rightarrow x z<y z .
$$

In any ordered field we define in a usual way an absolute value, $|x|$, and a limit of sequence, $\lim _{n \rightarrow \infty} a_{n}$. Note, however, that while in real analysis the formula $\forall \varepsilon>0$ stands for $\forall \varepsilon \in \mathbb{R}_{+}$, in an ordered field $(\mathbb{F},+, \cdot, 0,1,<)$ it means $\forall \varepsilon \in \mathbb{F}_{+}$.

The term $n$ is defined by

$$
n={ }_{d f} \underbrace{1+1+\ldots+1}_{n-\text { times }},
$$

while $\frac{n}{m}={ }_{d f} n \cdot m^{-1}$. On this basis we assume that any ordered field includes natural numbers $\mathbb{N}$ and rational numbers $\mathbb{Q}$.

We define the following subsets of $\mathbb{F}$ :

$$
\begin{aligned}
& \mathbb{L}=\{x \in \mathbb{F}:(\exists n \in \mathbb{N})(|x|<n)\}, \\
& \mathbb{A}=\left\{x \in \mathbb{F}:(\exists n \in \mathbb{N})\left(\frac{1}{n}<|x|<n\right)\right\}, \\
& \Psi=\{x \in \mathbb{F}:(\forall n \in \mathbb{N})(|x|>n)\}, \\
& \Omega=\left\{x \in \mathbb{F}:(\forall n \in \mathbb{N})\left(|x|<\frac{1}{n}\right)\right\} .
\end{aligned}
$$

The elements of these sets are called limited, assignable, infinitely large, and infinitely small numbers respectively. Here are some obvious relationships between these kinds of elements, we will call them $\Omega \Psi$ rules,

$$
\begin{aligned}
& (\forall x, y \in \Omega)(x+y \in \Omega, x y \in \Omega), \\
& (\forall x \in \Omega)(\forall y \in \mathbb{L})(x y \in \Omega), \\
& (\forall x)\left(x \in \mathbb{A} \Rightarrow x^{-1} \in \mathbb{A}\right), \\
& (\forall x \neq 0)\left(x \in \Omega \Leftrightarrow x^{-1} \in \Psi\right) .
\end{aligned}
$$

Referring to the set $\Omega$, an equivalence relation is defined by

$$
x \approx y \Leftrightarrow x-y \in \Omega .
$$

We say that $x$ is infinitely close to $y$, when the relation $x \approx y$ holds.

Although we present the above relations within the modern framework, all of them were explicitly discussed in [30, ch. 3]. 


\subsection{Archimedean axiom}

Here are some equivalent forms of the Archimedean axiom:

(A1) $(\forall x, y \in \mathbb{F})(\exists n \in \mathbb{N})(0<x<y \Rightarrow n x>y)$,

(A2) $(\forall x \in \mathbb{F})(\exists n \in \mathbb{N})(n>x)$,

(A3) $\lim _{n \rightarrow \infty} \frac{1}{n}=0$,

(A4) $(\forall x, y \in \mathbb{F})(\exists q \in \mathbb{Q})(x<y \Rightarrow x<q<y)$,

(A5) For any Dedekind cut $(A, B)$ of $(\mathbb{F},<)$ obtains $^{11}$

$$
(\forall n \in \mathbb{N})(\exists a \in A)(\exists b \in B)\left(b-a<\frac{1}{n}\right),
$$

(A6) $\Omega=\{0\}$.

Versions A1 and A2 are well-known, both in the mathematical as well as the historical context. In calculus courses, A3 is usually presented as a theorem rather than an axiom, however the Archmimedean axiom follows from some versions of the continuity of real numbers, or is explicitly included in other versions (see section 3.2. below). A6 reveals that in a non-Archimedean field the set of infinitesimals $\Omega$ contains at least one positive element, say $\varepsilon$. Then, by $\Omega \Psi$ rules, $\frac{\varepsilon}{n}$, as well as, $n \cdot \varepsilon$ are also infinitesimals.

The versions A1 to A6 above are equivalent within the framework of an ordered field while some of them, for instance A1, apply to an ordered group $(\mathbb{G},+,<)$. Then, the term $n x$ is defined by

$$
n x=d f \underbrace{x+x+\ldots+x}_{n-\text { times }} .
$$

We can also apply versions A4 and A5, provided the concept of fraction is interpretable in a group. Versions A3 and A6 involve the concept of an absolute value. While the very definition makes sense in any ordered group, some properties of the absolute value, such as $|x \cdot y|=|x| \cdot|y|$, require the order to be compatible both with sums and products. Hence, these versions need to be applied carefully.

At the end of the 19th century, a few non-Archimedean structures were introduced, however they contained rather exotic mathematical entities that provoked distrust. ${ }^{12}$ We present a non-Archimedean group made up

${ }^{11}$ For the remainder, a pair $(A, B)$ of non-empty sets is a Dedekind cut of a totally ordered set $(X,<)$ iff: (1) $A \cup B=X,(2)(\forall x \in A)(\forall y \in B)(x<y)$.

$12[27]$ provides a thorough overview of these structures. 
of then well-known objects, namely complex numbers; the simplicity of the model makes us wonder why it was not involved in the dispute concerning infinitesimals.

Let $(\mathbb{C},+, 0, \prec)$ be the additive group of complex numbers with the lexicographical order, i.e.,

$$
a+b i \prec c+d i \Leftrightarrow a<c \vee(a=c, b<d) .
$$

The order is compatible with sums, although not with products. One can easily show that $0 \prec i \prec 1$, moreover, for every natural number $n$ the inequality $n i \prec 1$ holds. The set $\{r i: r \in \mathbb{R}\}$ includes infinitesimals of the group $(\mathbb{C},+, 0, \prec){ }^{13}$

\subsection{Real numbers}

The field of real numbers is a commutative ordered field $(\mathbb{F},+, \cdot, 0,1,<)$ in which every Dedekind cut $(L, U)$ of $(\mathbb{F},<)$ satisfies the following condition:

$$
(\exists x \in \mathbb{F})(\forall y \in L)(\forall z \in U)(y \leq x \leq z) .
$$

Throughout the paper, we consider the condition $\mathrm{C} 1$ the Dedekind cut principle. Here are some other equivalent forms of $\mathrm{C} 1$ :

(C2) If $A \subset \mathbb{F}$ is a nonempty set which is bounded above, then there exists $a \in \mathbb{F}$ such that $a=\sup A$.

(C3) The field is Archimedean and every Cauchy (fundamental) sequence $\left(a_{n}\right) \subset \mathbb{F}$ has a limit in $\mathbb{F}$.

(C4) The field is Archimedean and if $\left\{A_{n} \mid n \in \mathbb{N}\right\} \subset \mathbb{F}$ is a family of descending, closed line segments, then $\bigcap_{n \in \mathbb{N}} A_{n} \neq \emptyset$.

Any equivalent form of $\mathrm{C} 1$ usually gets the name of continuity or completeness, and then the real numbers system is called the continuous ordered field or the complete ordered field. The version $\mathrm{C} 2$ is also known as Dedekind completeness or the least upper bound (LUB) principle, whereas the second part of C3 is called Cauchy completeness. Since Dedekind and Cauchy completeness are not equivalent, we prefer to use more specific names like Dedekind cut or LUB principle.

${ }^{13}[4]$ provides historical account of the Archimedean axiom, from Euclid and Archimedes, through Heiberg's edition of Greek text, to the 19th century theories of magnitudes developed by Stolz, Weber, Hölder and others. 
The above definition is based on the so called categoricity theorem which states that every two ordered fields that satisfy $\mathrm{C} 1$ are isomorphic. In that sense, the field of real numbers is the unique complete ordered field.

When dealing with non-Archimedean fields, the following theorem is of crucial importance: The field of real numbers is the biggest Archimedean field, that is, for any Archimedean field $(A,+, \cdot, 0,1,<)$, there is a subfield of the field of real numbers that is isomorphic to $(A,+, \cdot, 0,1,<)$. As a result, any field extension of the system of real numbers is a non-Archimedean field and includes infinitely many infinitesimal numbers. Below we present such an extension, namely the field of hyperreals $\left(\mathbb{R}^{*},+, \cdot, 0,1,<^{*}\right)$.

One way to obtain hyperreals is by the ultrapower construction. Here is a sketch of that approach. ${ }^{14}$ Let $(\mathbb{R},+, \cdot, 0,1,<)$ be the field of real numbers, let $\mathcal{U}$ be a nonprincipal ultrafilter on $\mathbb{N}$. The set $\mathbb{R}^{*}$ is defined as the quotient class of $\mathbb{R}^{\mathbb{N}}$ with respect to the following relation

$$
\left(r_{n}\right) \equiv\left(s_{n}\right) \Leftrightarrow\left\{n \in \mathbb{N}: r_{n}=s_{n}\right\} \in \mathcal{U},
$$

thus, $\mathbb{R}^{*}=\mathbb{R}^{\mathbb{N}} / \mathcal{u}$. New sums and products are defined pointwise, while the total order is defined by

$$
\left[\left(r_{n}\right)\right]<^{*}\left[\left(s_{n}\right)\right] \Leftrightarrow\left\{n \in \mathbb{N}: r_{n}<s_{n}\right\} \in \mathcal{U} .
$$

Hence, the product of hyperreal $\left[\left(r_{1}, r_{2}, \ldots\right)\right]$ and $\left[\left(s_{1}, s_{2}, \ldots\right)\right]$ gives $\left[\left(r_{1}\right.\right.$. $\left.\left.s_{1}, r_{2} \cdot s_{2}, \ldots\right)\right]$, and the relation $\left[\left(r_{1}, r_{2}, \ldots\right)\right]<^{*}\left[\left(s_{1}, s_{2}, \ldots\right)\right]$ holds when, for example, the set $\left\{n \in \mathbb{N}: r_{n}<s_{n}\right\}$ equals $\mathbb{N}$ minus some finite set (though the definition of order $<^{*}$ also includes other cases).

Standard real number, $r \in \mathbb{R}$, is represented by the class $[(r, r, r, \ldots)]$, i.e., the class of a constant sequence $(r, r, r, \ldots)$. Owing to these definitions, we employ the same symbols for real numbers in the standard and nonstandard context; we will also employ the same symbols for sums, products and order relation in the standard and non-standard context.

The equivalence class of the sequence $\left(\frac{1}{n}\right)$, i.e., the hyperreal number $\varepsilon=\left[\left(\frac{1}{n}\right)\right]$, is a model example of infinitesimal. As another infinitesimal, let us consider a hyperreal $\delta$ represented by the sequence $\left(\frac{1}{n^{2}}\right)$, that is $\delta=\left[\left(\frac{1}{n^{2}}\right)\right]$. It is easy to check that $0<\delta<\varepsilon$.

To study products of infinitely small and infinitely large numbers, let us define two infinite numbers,

${ }^{14}$ For details, see $[3,2]$. 


$$
K=[(n)]=[(1,2,3, \ldots)], \quad L=\left[\left(n^{2}\right)\right]=[(1,4,9, \ldots)] .
$$

Since products are defined pointwise, we easily obtain the following equalities

$$
K \cdot \varepsilon=1, \quad L \cdot \varepsilon=K, \quad K \cdot \delta=\varepsilon .
$$

Although $K$ and $L$ are not Cantor's ordinal numbers, these results undermine the seemingly obvious supposition that the product of an infinitely large and infinitely small number has to be infinitesimal regardless of the framework. In fact, within the field of hypereals we can realize all three options: the product can be an appreciable, infinitely large, or infinitely small number.

As already mentioned, in his argument Cantor employs the specific interpretation of the Archimedean property. Namely, instead of sums, he allows for multiplications. ${ }^{15}$ Adopting that perspective, axiom A1 will take the following form

$\left(A 1^{*}\right)\left(\forall x, y \in \mathbb{R}^{*}\right)\left(\exists n \in \mathbb{N}^{*}\right)(0<x<y \Rightarrow n \cdot x>y)$,

where the set of hypernatural numbers $\mathbb{N}^{*}$ is defined by

$$
\mathbb{N}^{*}=\left\{\left[\left(n_{j}\right)\right] \in \mathbb{R}^{*}:\left(n_{j}\right) \in \mathbb{N}^{\mathbb{N}}\right\} .
$$

The field of hyperreals is non-Archimedean in the sense of A1, yet it is Archimedean in the sense of $A 1^{*}$. Indeed, for any positive hyperreal numbers $x=\left[\left(r_{1}, r_{2}, r_{3}, \ldots\right)\right], y=\left[\left(s_{1}, s_{2}, s_{3}, \ldots\right)\right]$, due to the Archimedean property of real numbers, there is a sequence of natural numbers $\left(n_{j}\right)$ such that $n_{j} \cdot r_{j}>s_{j}$. Thus, the hypernatural number $K$ defined by $K=\left[\left(n_{j}\right)\right]$ is such that $K \cdot x>y$.

Finally, let us note that the set of assignable hyperreals $(\mathbb{A},+,<)$ is Archimedean in the sense A1, A2, A5, and non-Archimedean in the sense $\mathrm{A} 4$, while versions $\mathrm{A} 3$ and $\mathrm{A} 6$ do not apply to this structure; $(\mathbb{A},+,<)$ is not an ordered group, not to mention an ordered field. Therefore to deal with the Archimedean property we need a broader algebraic context.

\footnotetext{
${ }^{15}$ Under some interpretations, Cantor's term $\zeta \nu$ stands for specific infinite sum; see [27]. Whether it is a product or infinite sum, Cantor's interpretation differs from our modern understanding of A1, as well as from the version of the Archimedean property introduced by [47]; see section 6 below.
} 


\section{Cantor's proof}

Cantor's proof was presented in his letter to Goldscheider (Cantor, 13 May, 1887). Its crucial part was also included in the letter to Weierstrass (Cantor, 16 May, 1887). Then the former letter was made into section VI of the paper [16]. Below, we present the Philip Ehrlich translation of [16, ch. VI, pp. 406-409]; numerals 1-11 as well as some Greek and German words in square brackets are added; to enhance the numbered sentences, we also changed the setting of original sections.

"You mention in your letter the question of actual infinitely small magnitudes [Grössen]. At several places of my works you will find expressed the opinion that this is impossible, i.e., they are self-contradictory in thought, and I already implied in my work "Foundations of a General Theory of Manifolds", p. 8, $\S 4$, even though still with a certain reserve, that a rigorous proof of this position could be derived from the theory of transfinite numbers. During this winter, the time was first found to express my ideas on this subject in the form of a formal proof. It concerns the theorem:

[1] Non-zero linear numbers $\zeta$ (i.e., numbers which may be regarded as bounded, continuous lengths of straight lines) which would be smaller than each arbitrarily small finite number do not exist, i.e., they contradict the concept of linear numbers.

The thought process of my proof is simply as follows:

[2] I proceed from the assumption of a linear magnitude [linearen Grössen] $\zeta$ which is so small that its $n$-fold product $\zeta \cdot n$ is less than unity for each whole number, and prove from the concept of linear magnitude with the help of certain propositions of transfinite number theory,

[3] that even when $\nu$ is an arbitrarily large transfinite ordinal (i.e., the order type of a well-ordered set) $\zeta \cdot \nu$ is smaller than any finite magnitude that is as small as you please.

[4] This means that $\zeta$ cannot be made finite through any actual infinite multiplication [Vervielfachung], and is therefore certainly not an element of finite magnitude.

[5] Thus, the assumption we made contradicts the concept of a linear magnitude, which is of the sort that, according to it each linear magnitude must be thought of as an integral part of another, in particular of finite linear magnitude. So nothing is left but to let go of the assumption that there is a magnitude $\zeta$ which for any finite whole number $n$ would be smaller than $\frac{1}{n}$, and with this our proposition has been proven. 
It seems to me that this is an important application of the theory of transfinite numbers, which is capable of pushing aside widespread prejudices.

[6] The fact of actual infinite numbers is thus so little ground for the existence of actual infinitely small magnitudes that, on the contrary, the impossibility of the latter can be proven with the former.

[7] I also don't believe that this result can be reached fully and strictly in any other way.

[8] The need of our theorem is especially clear for the purpose of opposing the newer attempts of O. Stolz and P. Dubois-Reymond to derive the legitimacy of actual infinitely small magnitudes from the so-called "Archimedean axiom" (cf. O. Stolz, "Zur Geometrie der Alten, insbesondere über ein Axiom des Archimedes" 1881-1882, 1883; "Die unendlich kleinen Grössen" 1884; "Vorlesungen über allgemeine Arithmetik", Part 1, Leipzig 1885, p. 205).

[9] Archimedes appears to be the first to remark that, the assertion used in Euclid's Elements, where upon from any arbitrarily small line segment can be produced through sufficiently large multiplication [Vervielfachung] an arbitrarily large line segment, requires proof, and for that reason he believed that this assertion should be called an "Assumption".

[10] (Cf. Euclid's Elements, Book V, Definition 4: Magnitudes are said to have a ratio to one another which are capable, when multiplied $\left[\pi \circ \lambda \lambda \alpha \pi \lambda \alpha \sigma \iota \alpha \zeta \delta^{\prime} \mu \varepsilon v \alpha\right]$, of exceeding one another; also, especially Elements, Book X, Proposition 1, Archimedes' The Sphere and Cylinder I, Postulate 5 and the Introduction to his work: The Quadrature of the Parabola).

[11] Now it is the reasoning of those authors (O. Stolz loc. cit.), that if one deletes this supposed "axiom", the permissibility of actual infinitely small magnitudes, which are there called "moments", would emerge.

[12] But if the above theorem of mine is applied to the continuous straight line, the necessity of the Euclidean assumption immediately follows".

\section{Magnitudes and Archimedean property in Greek mathematics}

Cantor's sentences [9] and [10] as well as the bracketed apposition in sentence [1] explicitly refer to Greek mathematics, therefore we dedicate this section to ancient versions of the Archimedean property. 
We start with a brief description of the interest in ancient mathematics prevalent in the second half of the 19th century. In the 1880s, Johan L. Heiberg published Archimedis opera Omnia [37] and then Euclid's Elements [38]. They are both arranged in the same format: Greek text and Latin translation authored by Heiberg are provided alternately page by page. For the mathematicians of that time, these were standard source books for Greek understanding of magnitude. Cantor, Peano, and Hilbert cite them when discussing Euclid and Archimedes. However, the reading of ancient texts hinges upon a philosophical disposition. While, for instance, Hilbert's Grundlagen der Geometrie provides an interpretation of Euclid's Elements, Cantor's comments on the Archimedean property and the concept of magnitude reveal his Platonic propensity and belief that with new definitions, he complements the Greek idea of continuum rather than introducing a new concept. The 19th-century renaissance of Greek mathematics, specifically mathematicians' interest in the concept of magnitude and the theory of proportion, was initiated by German mathematician and historian Hermann Hankel. In 1874's Zur Geschichte der Mathematik in Altertum und Mittelalter, he developed modern formalizations of books V and VI of the Elements, and his symbolic representations of Euclid schematic phrases were then adopted in Heiberg's Latin translation. ${ }^{16}$

We must also note Hermann Grassmann's Lehrbuch der Arithmetik, published 1861. It was the first monograph dedicated to totally ordered groups. The idea of an order compatible with sums was employed in every axiomatic characteristic of a magnitude of that time, whereas Dedekind employed the idea of a total order compatible with sums and products in his definition of rational and real numbers, as developed in [23].

\subsection{Book V of Euclid's Elements}

The term linear number as it occurs in the sentence [1] refers to a closed line segment. The bracketed original German phrase reads: d.h. kurz gesagt, solche Zahlgrößen, welche sich unter dem Bilde begrenzter geradliniger stetiger Strecken vorstellen lassen. Thus, linear numbers are to represent - unter dem Bilde [...] vorstellen lassen - closed line segments, the model example of ancient Greek magnitudes.

${ }^{16}$ See [35, pp. 389-404]. 
To elaborate, the general term $\mu \varepsilon^{\prime} \gamma \varepsilon \vartheta$ os covers line segments, triangles, convex polygons, circles, solids, angles, and arcs of circles. We formalize (write down in symbols) Euclid's magnitudes of the same kind (line segments being of one kind, triangles being of another, etc.) as an additive semigroup with a total order, $(M,+,<)$, characterized by the following five axioms:

$$
\begin{aligned}
& \text { E1 }(\forall x, y)(\exists n \in \mathbb{N})(n x>y), \\
& \text { E2 }(\forall x, y)(\exists z)(x<y \Rightarrow x+z=y), \\
& \text { E3 }(\forall x, y, z)(x<y \Rightarrow x+z<y+z), \\
& \text { E4 }(\forall x)(\forall n \in \mathbb{N})(\exists y)(x=n y), \\
& \text { E5 }(\forall x, y, z)(\exists v)(x: y:: z: v) .
\end{aligned}
$$

The total order, both in book $\mathrm{V}$ as well as throughout the Elements, is a primitive notion characterized by transitivity and the trichotomy law. Unlike modern mathematics, in Greek mathematics it is applied not only to line segments, but to figures at all, e.g. in proposition I.6, triangles are compared in terms of lesser-greater.

E1 is Euclid definition 4 of book V. The sign :: represents proportion as provided in definition 5 of book $\mathrm{V}$. The fact that Greek line segments are closed segments is explicated in definition 3 of book I, which reads: "And the extremities of a line are points". Moreover, all throughout the Elements, line segments are represented by their end-points, such as A, B, whether in the text as $\mathrm{AB}$, or on diagrams, when $\mathrm{A}, \mathrm{B}$ stand next to intersections of lines, or next to short vertical lines depicting the ends of segments; for instance, all throughout book V magnitudes are represented by line segments with short, bounding vertical lines.

To be clear, we do not suggest that Cantor based his argument on such exegesis. Our point is that over the course of history, from ancient to modern times, line segments were considered what we now call closed line segments, i.e., segments with their ends. In the second half of the 19 th century, when the idea of totally ordered sets was introduced, it made distinguishing between closed and open line segments easy. These new concepts launched the mathematical career of open segments.

Throughout the ages, essentially owing to Descartes and Euler, the structure of Greek magnitudes $(M,+,<)$ was transformed into an ordered field $(M,+, \cdot, 0,1,<)$, and then, a number line. In the late 19th century, the number line was turned into the continuous line of real numbers. In 
this way, Cantor's sentence [12] refers to the 19th century characteristics of real numbers, rather than the ancient Greek structure of line segments.

In translations, the Greek word $\mu \varepsilon ́ \gamma \varepsilon \vartheta o \varsigma$ has been rendered in Latin as quantitas, in English as quantity or magnitude, in French - as quantité or grandeur, in German - as Quantität, or Grösse. In the 20th century mathematics, the term magnitude was slowly replaced with real numbers; still, in the late 40s, Nicolas Bourbaki used the term grandeur meaning real numbers. ${ }^{17}$ In [14], the term Zahlengrössen stands for what we consider to be real numbers, while already in [17] the term reellen Zahlen occurs. ${ }^{18}$ [23] defines real numbers as all rational and irrational numbers: aller reellen, d.h. aller rationalen und irrationalen Zahlen.

In sentence [10], Cantor cites the Greek text of definition V.4, however, in sentences [4] and [9], he interprets the word $\pi 0 \lambda \lambda \alpha \pi \lambda \alpha \sigma \iota \alpha \sigma \mu o ́ s$ as Vervielfachung (multiplication). Euclid's $\pi \circ \lambda \lambda \alpha \pi \lambda \alpha \sigma \iota \alpha \sigma \mu o ́ s ~ m e a n s ~ m u l t i-$ plicity rather than multiplication. Whereas multiplicity of a magnitude $x$ means the reiterated addition of that magnitude, that is $x+\ldots+x$, there was no multiplication of any kind in Greek geometry. Although the reading $n x=x+\ldots+x$ was standard at that time, Cantor adopted a specific interpretation of the Archimdean property, namely, at first, instead of $n x$ he assumed $n \cdot x$, then in the place of $(\forall n \in \mathbb{N})$, he allowed $(\forall \nu \in O r d)$.

In section 3.2 above, we have shown that by the same kind of interpretation, when instead of $n x$ we take $n \cdot x$, and then change the range of the variable $n$ from $\mathbb{N}$ to $\mathbb{N}^{*}$, we reach the conclusion that the field of hyperreal numbers is Archimedean in the modified sense $A 1^{*}$, although it is non-Archimedean in the standard sense A1.

\subsection{Archimedean property}

There are two versions of the Archimedean property in the Opera omnia [37]. In the treaty On spiral lines, Archimedes applies Euclid's version E1, although he calls it Lemma. In On the sphere and cylinder, the Lemma reads:

"Further, of unequal lines, unequal surfaces, and unequal solids, the greater exceeds the lesser by such a magnitude, as when added to itself, can be

${ }^{17}$ See. [10].

${ }^{18}$ See Section 7 below. 
made to exceed any assigned magnitude among those which are comparable with and one another". ${ }^{19}$

We formalize it with the following formula:

$$
\text { LA }(\forall x, y, z)(\exists n \in \mathbb{N})(x<y \rightarrow n(y-x)>z) \text {. }
$$

This version is placed among four lemmas, such as e.g. the following one:

"Of all lines which have the same extremities the straight line is the least". ${ }^{20}$

Clearly, Archimedes could not prove his lemmas. In fact, there is no mention throughout the treaty that any proof was needed at all. Yet, when, in the Quadrature of the parabola he reiterates the LA version, he also adds this comment:

"The earlier geometers have also used this lemma; for it is by the use of this same lemma that they have shown that circles are to one another in the duplicate ratio of their diameters, and that spheres are to one another in the triplicate ratio of their diameters, and further that every pyramid is one third part of the prism which has the same base with the pyramid and equal height; also, that every cone is one third part of the cylinder having the same base as the cone and equal height they proved by assuming a certain lemma similar to that aforesaid. And, in the result, each of the aforesaid theorems has been accepted no less than those proved without the lemma". ${ }^{21}$

The results mentioned in this passage are Euclid's propositions XII.2, XII.18, XII.7, and XII.10 respectively. Archimedes, thus, evokes the authority of Euclid to justify his reference to LA. Cantor could have considered Archimedes' restraint revealed in these lines when he wrote: "Archimedes appears to be the first to remark that, the assertion used in Euclid's Elements [...] requires proof".

Viewed from the mathematical perspective, there were no other axioms, lemmas or definitions in Greek mathematics allowing to deduce the Archimedean property. Book V of the Elements encapsulates all Greek science of magnitudes, while in our axiomatic account of the theory, E1 is an independent axiom.

\footnotetext{
$19[36$, p. 4].

20 [36, p. 3].

21 [36, p. 234].
} 
Archimedes was the unsurpassed champion of the exhaustion method, he also contributed to the foundations of mathematics by expanding the scope of the concept of magnitude to curve lines and surfaces. Nevertheless, he certainly did not seek to prove the LA lemma.

To sum up, Cantor's note in sentence [9] on Archimedes' will to prove LA reflects his attitude towards the Archimedean property rather than historical facts.

Finally, let us note that it was Stolz who coined the name Archimedean axiom. He referred to the lemma as presented in On the sphere and cylinder and Quadratura of the parabola as a model version of the axiom. ${ }^{22}$ The name prevailed in mathematics due to his often-cited books Vorlesungen über Allgemeine Arithmetik and Theoretische Arithmetik. On the other hand, Heiberg, in his comment on the Archimedean lemma, cites Euclid's definition V.4 and notes that these two are the same axiom. ${ }^{23}$ This comment of Heiberg, it seems, confirmed the name Archimedean axiom for Euclid's definition V.4.

\section{Theory of magnitudes in the late 19th century}

In sentences [8] and [11] Cantor refers to Stolz's idea of introducing nonArchimedean numbers via axioms for magnitudes, while sentences [9] and [10] contain comments on Archimedes and Euclid. In fact, it was Stolz who confirmed mathematical studies of the concept of magnitude, as opposed to historical studies. Although many names were involved in this process, we present the mathematical extracts of the concept of magnitude as developed in $[25,26,47,49,43]$. This is how the movement has been characterized by Hölder's 1901 [43]:

"The theory of measurable magnitudes was developed to a high level by Euclid. Recently, it has been treated in depth from a number of different points of view. Nevertheless, it seems that the theory has not been treated exhaustively; further, errors and obscurities have appeared in some of the more recent treatments. This is why I think that a reformulation of this important and fundamental theory will be profitable". ${ }^{24}$

\footnotetext{
${ }^{22}$ See [47, pp. 70, 332].

${ }^{23}$ See [37, p. 11].

$24[44$, p. 238]. It is an English translation of [43] by J. Mitchell.
} 
In a way, [43] finished and crowned these studies. In 1899, in the first edition of his Grundlagen der Geometrie, Hilbert provided axioms for an ordered field. Then, in [41], he provided the first ever axioms for real numbers. From that moment on, the mathematical studies of the concept of magnitude were redirected to axioms for real numbers, the continuity axiom specifically.

In what follows, we present symbolic accounts of the concept of magnitude. To be clear, no one in the 19th century applied such symbols. We decided on that form of presentation to clarify the mathematical background of Cantor's considerations. The common feature of all of these accounts was that the structure of magnitudes is a totally ordered, semigroup $\mathfrak{M}=(M,+,<)$ equipped with different axioms depending on the author. $^{25}$

\subsection{Du Bois-Reymond, 1882/1887}

B1 $(\forall x)(\exists y, z)(y<x \wedge z>x)$,

B2 $(\forall x, y)(x+y>x)$,

B3 $(\forall x)(\forall n \in \mathbb{N})(\exists y) x=n y)$,

B4 $(\forall x, y)(\exists z)(x<y \Rightarrow x+z=y)$,

B5 $(\forall x, y, z)(x<y \Rightarrow x+z<y+z)$,

B6 $(\forall x, y)(\exists n \in \mathbb{N})(x \leq y \Rightarrow n x \geq y)$,

B7 $(\forall x, y)(\forall n \in \mathbb{N})(\exists z)(z<y \wedge x=n z)$.

\subsection{Otto Stolz, 1885}

S1 $(\forall x, y)(\exists n \in \mathbb{N})(x<y \Rightarrow n x>y)$,

S2 $(\forall x, y)(\exists ! z)(x<y \Rightarrow x+z=y)$,

S3 $(\forall x, y, z)(x<y \Rightarrow x+z<y+z)$,

$\mathrm{S} 4(\forall x, y)(x+y>x)$,

S5 $(\forall x)(\forall n \in \mathbb{N})(\exists y)(x=n y)$.

\subsection{Heinrich Weber, 1895}

W1 $(\forall x, y)(\exists n \in \mathbb{N})(n x>y)$,

${ }^{25}$ For details, see [1]. 
W2 $(\forall x, y)(\exists z)(x<y \Rightarrow x+z=y)$,

W3 $(\forall x, y)(x+y>x)$,

W4 The order $<$ is dense and for every Dedekind cut $(A, B)$ of $(M,<)$, obtains

$(\exists z)(\forall x \in A)(\forall y \in B)(x \leq z \leq y)$.

\subsection{Otto Hölder, 1901}

Hölder clarifies the concept of addition by following axioms:

$$
\begin{aligned}
& M \times M \ni(x, y) \mapsto x+y \in M . \\
& (\forall x, y, z)[(x+y)+z=x+(y+z)] .
\end{aligned}
$$

Specifically, he does not assume the commutativity of addition. As regards the order $<$, his only assumption is the so called trichotomy law, namely

For every two elements $x, y$, one and only one of the three possibilities obtains: $x<y \vee x=y \vee x>y$.

Here are his axioms.

H1 $(\forall x)(\exists y)(y<x)$,

H2 $(\forall x)(\forall y)(x+y>x \wedge x+y>y)$,

H3 $(\forall x, y)(\exists z, w)(x<y \Rightarrow(x+z=y \wedge w+x=y))$,

H4 For every Dedekind cut $(A, B)$ of the set $(M,<)$, obtains $(\exists z)(\forall x \in A)(\forall y \in B)(x \leq z \leq y)$.

Hölder managed to show that axioms H1-H4 entail the transitivity of the order $<$, the commutativity of addition, and above all, the Archimedean property.

\subsection{Non-Archimedean group}

As we can see, Du Bois-Reymond, Stolz, and Weber explicitly assume the Archimedean property. These are axioms B6, S1, and W1 respectively. When adopting their perspective, rejection of the Archimedean property does not imply inconsistency; we could say it would lead to a concept of a non-Archimedean ordered group.

Yet, in sentence [12], Cantor claims: "if the above theorem of mine is applied to the continuous straight line, the necessity of the Euclidean assumption immediately follows". These are Hölder's results that could 
support this belief, rather than Cantor's alleged theorem given in sentence [3]. Instead of infinitesimals, "continuous straight line" proved to be the key concept in these considerations. However, Cantor never adopted Dedekind's version of continuity.

\section{Cantor's continuum}

Neither Cantor, nor Dedekind was quite sure whether their versions of continuity of real numbers were equivalent. It was partly because there were no obvious framework that would enable to establish or dismiss the equivalence of Cantor's and Dedekind's versions of continuity. While Dedekind Cut Principle applies to totally ordered sets, Cantor sought for universal formula which could be applied in any framework. In fact, he considered an ordered field, a metric space and a totally ordered set. In each context, he tried to apply his newly discovered idea of derived set, $P^{\prime}$.

Nevertheless, both Cantor and Dedekind developments had a clear reference object: Euclid's geometrical line.

\subsection{Cantor on the field of real numbers}

In [14], real numbers are made up of fundamental sequences (Cauchy sequences). Cantor managed to define field operations as well as the total order of real numbers. Dealing with numbers, he applies the concept of sequence limit. From the perspective of the continuity of real numbers, the following sentence is crucial:

"While domains B and A are so related, that although each $\mathbf{a}$ is assigned to a certain $\mathbf{b}$, but not each $\mathbf{b}$ can be assigned to $\mathbf{a}$, it turns out that both $\mathbf{b}$ can be assigned to a certain $\mathbf{c}$, and each $\mathbf{c}$ can be assigned to a certain b". ${ }^{26}$

The phrase "each a is assigned to a certain b" means that each rational number can be represented as a Cauchy sequence of rational numbers. The phrase "not every $\mathbf{b}$ can be assigned to any $\mathbf{a}$ " means that the space of rational numbers is not Cauchy-complete. The phrase "each c can be assigned to a certain b" means that the space of real numbers is Cauchycomplete. It could be rendered as follows: $A^{\prime}=B, B^{\prime}=B$, where prime

${ }^{26}$ ([14, p. 95]. Letters a, b, c stand for elements of the sets A, B, C respectively. 
represents Cantor's derivative set. Cantor does not prove any of these claims and - as far as we know - never returned to this issue.

At the end of section $\S 1$, Cantor declares that he develops the results of Book X of Euclid Elements. Interestingly, while integers and rational numbers are called Zahlen, for real numbers Cantor adopted the term Zahlengrössen, which could mean numbers assigned to magnitudes.

In the next section, Cantor introduces his famous axiom relating real numbers and geometric line. First, he shows how to assign for a point on the straight line a real number. To this end, he determines a unit segment and assumes that any point on the line is in a rational (rationales Verhältnis) or irrational (im andern Falle) ratio to the unit. Due to this very assumption he is really in the heir to Euclid, specifically in his understanding of commensurable and in-commensurable line segments as presented in Book X of the Elements. ${ }^{27}$

Then Cantor writes:

"To make complete the relationship of the domain of number magnitudes [Zahhlengrössen] defined in $\S 1$ with straight line geometry outlined in this $\S$, I should only add an axiom, which is simply the converse, to every number magnitude there corresponds a definite point of the line whose coordinate is equal to that number magnitude, and equal in the sense as explained herein $\S$. I call this statement an axiom, because it is in its nature that it is not generally provable". ${ }^{28}$

When points on the straight line and real numbers are identified, Cantor continues to study subsets of the line. Within the geometrical context, he prefers to apply the derivative set $P^{\prime}$ of a set $P$, rather than the concept of sequence limit, as defined in an ordered field. In fact, to define $P^{\prime}$ one only needs a structure of open line segments, thus the idea of $P^{\prime}$ can be transferred to a totally ordered set $(X,<)$.

\subsection{Correspondence with Dedekind}

In $[23, \S 3]$, Dedekind coined his cut principle as the "essence of continuity". The Preface mentions [14] and reads:

${ }^{27}$ In terms of the unit segment, Cantor belongs to the tradition which goes back to [24]. There was, of course, no universal unit segment in Greek mathematics.

${ }^{28}$ [14, p. 97]. As for the last sentence, both Cantor's axioms turned out to be theorems within the framework of axiomatic account of Euclid's geometry; see $[9, \S 20]$ or $[34, \S 21]$. 
"After a hasty reading, it seems to me that the axiom given in Section II of that paper (except for the form of presentation) agrees with what I designate in Section III as the essence of continuity". ${ }^{29}$

Dedekind seems never had a time to study Cantor's paper in depth and decide whether their axioms really agree.

In the letter to Dedekind dated May 17, 1887, Cantor raised the objection that the cut principle applies both to integers and to real numbers: "this property also holds of the system of all integers". ${ }^{30}$ In the Post Scriptum, he reiterated his objection by writing:

"you lay special emphasis on IV [i.e. the cut principle] because this property distinguishes the complete domain of numbers from the domain of all rational numbers; however it seems to me for the above reasons that one cannot give property IV the name essence of continuity". ${ }^{31}$

On May 17, 1887, Dedekind replied: "you worry that my exclusive stressing of IV as the property in which the essence of continuity is expressed could lead to misunderstanding. I do not share this concern". ${ }^{32}$ Then he adds that the cut principle is the essence of continuity when applied to a dense total order.

Cantor refers to Dedekind's reply in the first sentences of the letter dated June 20, 1887. It reads:

"Thank you for your letter of 18 May. I completely agree with its contents; and I acknowledge that the difference in our opinion of view was merely external". 33

In the rest of the letter Cantor presents his proof to the effect subsets of $\mathbb{R}^{2}$ can be in one-to-one relation with "continuous line". ${ }^{34}$

The standard reading of that exchange is that Cantor simply misinterpreted Dedekind by applying the cut principle to a totally ordered set, rather than to a densely ordered set. Nevertheless in $[15, \S 9]$, Dedekind's

\footnotetext{
$29[31$, p. 767$]$.

$30[31$, p. 852$]$.

31 [31, p. 852]. [23, § 2] provides a characteristic of the total order of rational numbers, $R$ in his notation, which we paraphrase as follows: I transitivity, II density, III every rational number determines Dedekind cut of the set $(R,<)$. The cut principle, the condition IV, occurs in section $\S 3$ called Continuity of the Straight Line.
}

$32[31$, p. 852$]$.

33 [31, p. 853].

$34[31$, p. 853$]$. 
construction of real numbers was perfectly summarized. Thus, there was no misinterpretation. The point is a philosophical question: the "essence of continuity". Doubts whether Dedekind's cut principle provides a universal characteristic of the continuum have been voiced already in $[15, \S 10]$. In fact, they have never been dispelled.

\subsection{Cantor on continuum in metric space}

$[15, \S 9]$ summaries some 19th century theories of real numbers, namely: Weierstrass', as developed in 1872' Kossak Die Elemente der Arithmetik, Dedekind's, as developed in [23], and Cantor's, as developed in [14].

$[15, \S 10]$ is dedicated to the continuum. Tracing back the history of this concept, Cantor discusses ancient Greek and Medieval philosophers. He believes that "the underlying idea has taken on different meanings". Therefore, his definition could be compared with, for instance, Aristotle's ex partibus sine fine divisibilibus. ${ }^{35}$ This very section initiated a branch of point-set topology, namely continuum theory, that is the study of compact and connected spaces. Yet Cantor's own definition is a bit different.

According to Cantor, a subset $T$ of the space $\mathbb{R}^{n}$ with Euclidean metric is connected if for any of its points $t_{0}$ and $t^{0}$ and any positive real number $\varepsilon$ there are finitely many points $t_{1}, t_{2}, \ldots, t_{n}$ of $T$ such that the distances $d\left(t_{0}, t_{1}\right), d\left(t_{1}, t_{2}\right), \ldots, d\left(t_{n}, t^{0}\right)$ are all less than $\varepsilon$.

$T$ is perfect if $T=T^{\prime}$, where

$$
x \in T^{\prime} \Leftrightarrow \lim _{n \rightarrow \infty} x_{n}=x,
$$

for some $\left(x_{n}\right) \subset \mathbb{R}^{n} \backslash\{x\}$; convergence of a sequence is defined in the metric space $\mathbb{R}^{n}$. The latter condition is equivalent to the following assertion: Every convergent series $\left(t_{n}\right) \subset \mathrm{T}$ has a limit in $T$, and for every $t \in T$, there exists a sequence $\left(t_{n}\right) \subset T$ such that $\lim _{n \rightarrow \infty} t_{n}=t$.

Then comes the famous definition:

"I therefore define a point-continuum inside $G_{n}\left[\mathbb{R}^{n}\right.$ in our notation $]$ as a perfect-connected". ${ }^{36}$

${ }^{35}$ It is a scholastic version of Aristotles characterization of magnitude ( $\left.\mu \varepsilon ́ \gamma \varepsilon \vartheta o \varsigma\right)$ as provided in Physics, VI: divisible into divisibles that are infinitely divisible. It can be show that Aristole's definition is compatible with Euclid's characteristic of a line segment; see [1].

${ }^{36}$ See [31, pp. 903-906]. 
Based on this definition, Cantor rebukes definition of the continuum as given in $[6, \S 38]$ and Dedekind's cut principle. He claims that nonconnected sets exemplify Bolzano's definition. ${ }^{37}$ As for Dedekind, Cantor writes:

"Likewise, it seems to me that in the article (Continuity and irrational numbers) only another property of the continuum has been one-sidedly emphasized, namely, that property which it has in common with all 'perfect' sets". 38

\subsection{Order type of linear continuum (Linearkontinuum)}

$[17, \S 11]$ provides the order type characteristic of the segment $[0,1]$ of numbers (reellen Zahlen) with their natural order (ihrer natürlichen Rangordung). ${ }^{39}$ Cantor proves that any linearly ordered set $(X,<)$ with the first and the last element, that is (1) perfect, and (2) contains a subset $A \subset X$ which is dense in $(X,<)$ and of cardinality $\aleph_{0}$ is isomorphic to the set $([0,1],<)$.

Since in 1883 Cantor interprets Dedekind Cut Principle as a property of perfect set, in 1895 he could be certain that Dedekind Cut Principle did not provide the "essence of continuity". Nowadays we can support his belief by a simple example. Namely, let $X=[0,1] \times[0,1]$, be the Cartesian product of real numbers segments with lexicographical order. The set $(X,<)$ is continuous in terms of the cut principle, however, it is not a separable space.

\section{Modern account of the product $\zeta \cdot \nu$}

In this section, we firstly provide an alternative arithmetic for Cantor's sums and products of ordinal numbers. Then, we introduce a non-Archimedean field $O N A G$ which includes the class of ordinal numbers, Ord. As the field $O N A G$ includes both ordinal and infinitesimal numbers, we can show that Cantor hypothesis concerning products of ordinal and infinitesimal numbers, as presented in sentence [3], fails.

\footnotetext{
${ }^{37}$ Indeed, Bolzano definition of the continuum boils to the fact that the continuum has no isolated points.

38 [31, p. 906].

${ }^{39}$ Cantor had never explained what natural order means in mathematical terms.
} 


\subsection{Normal sums and products of ordinal numbers}

Let us start with a remainder of the normal form theorem [18]: For every ordinal number $\alpha \in$ Ord, there are ordinal numbers $\eta_{1}, \ldots, \eta_{h}$, and natural numbers $h, p_{i} \in \mathbb{N}$ such that

$$
\alpha=\omega^{\eta_{1}} \cdot p_{1}+\ldots+\omega^{\eta_{h}} \cdot p_{h},
$$

where $\eta_{1}>\ldots>\eta_{h}$.

This representation of $\alpha$ is unique. Moreover, it is finite, due to the assumption concerning the index $h$.

Based on this theorem, [39] introduced the so-called normal sums and products of ordinal numbers. Namely, for

$$
\alpha=\omega^{\eta_{1}} \cdot p_{1}+\ldots+\omega^{\eta_{h}} \cdot p_{h}, \quad \beta=\omega^{\eta_{1}} \cdot q_{1}+\ldots+\omega^{\eta_{h}} \cdot q_{h}
$$

their normal sum $+_{n}$ and normal product $\cdot_{n}$ is defined by ${ }^{40}$

$$
\begin{aligned}
\alpha+{ }_{n} \beta & ={ }_{d f} \quad \omega^{\eta_{1}} \cdot\left(p_{1}+q_{1}\right)+\ldots+\omega^{\eta_{h}} \cdot\left(p_{h}+q_{h}\right) \\
\alpha \cdot{ }_{n} \beta & ={ }_{d f} \sum_{1 \leq i, j \leq h} \omega^{\eta_{i}+{ }_{n} \eta_{j}} \cdot p_{i} q_{j}
\end{aligned}
$$

Contrary to Cantor's sums and products of ordinal numbers, normal sums and products are commutative and compatible with the standard order of ordinal numbers, that is

$$
\begin{gathered}
\alpha+{ }_{n} \beta=\beta+{ }_{n} \alpha, \quad \alpha \cdot{ }_{n} \beta=\beta \cdot{ }_{n} \alpha, \\
\alpha<\beta \Rightarrow \alpha+{ }_{n} \gamma<\beta+{ }_{n} \gamma, \quad \alpha<\beta \Rightarrow \alpha \cdot{ }_{n} \gamma<\beta \cdot{ }_{n} \gamma .
\end{gathered}
$$

Thus, the structure $\left(\operatorname{Ord},+_{n},{ }_{n}, 0,1,<\right)$ is an abelian semigroup.

Hence, e.g. since $\omega=\omega \cdot 1+0$, and $1=\omega \cdot 0+1$, we calculate the normal sums of $\omega+_{n} 1$ and $1+{ }_{n} \omega$ as follows,

$$
\begin{aligned}
& 1+{ }_{n} \omega=(\omega \cdot 0+1)+{ }_{n}(\omega \cdot 1+0)=\omega \cdot(0+1)+1=\omega+1, \\
& \omega+{ }_{n} 1=(\omega \cdot 1+0)+{ }_{n}(\omega \cdot 0+1)=\omega \cdot(1+0)+1=\omega+1 .
\end{aligned}
$$

Similarly, we calculate

$$
\begin{aligned}
& 2 \cdot{ }_{n} \omega=(\omega \cdot 0+2) \cdot{ }_{n}(\omega \cdot 1+0)=\omega^{2} \cdot 0+\omega \cdot 2+0=\omega \cdot 2, \\
& \omega \cdot{ }_{n} 2=(\omega \cdot 1+0) \cdot{ }_{n}(\omega \cdot 0+2)=\omega^{2} \cdot 0+\omega \cdot 2+0=\omega \cdot 2 .
\end{aligned}
$$

As is well known, in Cantor's arithmetic the inequalities hold $1+\omega<$ $\omega+1$, and $2 \cdot \omega<\omega \cdot 2$.

\footnotetext{
${ }^{40} \mathrm{We}$ assume for the use of the definition, that some $p_{i}$ or $q_{i}$ could equal 0 .
} 


\subsection{Conway numbers}

$[20,21]$ introduces a very special non-Archimedean ordered field; it is usually called the field of surreal numbers or in short ONAG (the acronym for on numbers and games). In fact, [28] proves that $(O N A G,+. \cdot, 0,1,<)$ is the biggest non-Archimedean field.

While Conway develops his theory beyond the framework of the set theory, [32] manages to rediscover surreal numbers in the set theory, and provides a suggestive representation. Namely, a surreal number is a function a from an ordinal $\alpha$ into the set $\{+,-\}$, that is

$$
\mathbf{a}: \alpha \mapsto\{+,-\} \text {. }
$$

Hence, every ordinal number $\alpha$ is represented by the $\alpha$-length string of pluses

$$
\alpha \sim(\underbrace{++\ldots}_{\alpha}) .
$$

To compare surreal numbers $\mathbf{a}, \mathbf{b}$ in terms of lesser-greater, when $\alpha<\beta$, where $\alpha$ and $\beta$ are domains of $\mathbf{a}, \mathbf{b}$ respectively, we make up the sequence $\mathbf{a}$ by $0 \mathrm{~s}$, to the sequence of $\beta$-length. Then, the total order $\mathbf{a}<\mathbf{b}$ is defined by lexicographical order, given

$$
-<0<+.
$$

For example,

$$
(--)<(-)<(-+)<(+)<(++-)<(++) .
$$

We can show that the field $O N A G$ includes the structure $\left(O r d,{ }_{n},{ }_{n}\right.$, $0,1,<)$. Therefore, within the framework of surreal numbers, Cantor's ordinal numbers are subject to field operations. Next to the ordinal number $\omega$, in the field $O N A G$, there are also elements such as

$$
-\omega, \quad \omega-1, \quad \frac{\omega}{2}, \quad \frac{1}{\omega} .
$$

Due to Gonshor's development, we can represent these numbers as follows

$$
\begin{aligned}
& -\omega=(\underbrace{--\ldots}_{\omega}), \\
& \omega-1=(\underbrace{+++\ldots}_{\omega}-),
\end{aligned}
$$




$$
\begin{aligned}
& \frac{\omega}{2}=(\underbrace{+++\ldots}_{\omega} \underbrace{---\ldots}_{\omega}), \\
& \frac{1}{\omega}=(\underbrace{+---\ldots}_{\omega}) .
\end{aligned}
$$

Since every infinite ordinal number $\alpha$ is an infinite element of the field $O N A G$, i.e. it is an element of the class $\Psi$, as defined in section 3 above, the element $\alpha^{-1}$ is infinitesimal. In this way, the field of surreal numbers provides a framework to test Cantor's hypothesis concerning the products of infinitesimal and ordinal numbers.

\subsection{Falsifying Cantor's hypothesis}

Sentence [3] includes the key mathematical part of Cantor's argument, we call it the Infinitesimals Hypotheses $(\mathrm{IH})$ : when $\zeta$ is infinitesimal and $\nu$ "is an arbitrarily large transfinite ordinal $[\ldots] \quad \zeta \cdot \nu$ is smaller than any finite magnitude", in symbols

$$
(\forall \nu \in O r d)(\zeta \cdot \nu<1) .
$$

Cantor had never defined the product of infinitesimal and ordinal numbers, especially he had never proved the claim IH. The framework of surreal numbers enables, both make sense of the product $\zeta \cdot \nu$, and falsify the claim IH.

For the falsification part, let $\zeta$ be a positive infinitesimal. Then $\zeta^{-1} \in$ $\Psi$, i.e. $\zeta^{-1}$ is infinitely large number in the field $O N A G$. Due to Gonshor's representation of surreal numbers, we can find and ordinal number $\alpha$ greater than $\zeta^{-1}$. By the standard rules of an ordered field, we have

$$
\zeta^{-1}<\alpha \Rightarrow \alpha^{-1}<\zeta .
$$

Similarly, by the standard rules of an ordered field

$$
\alpha^{-1}<\zeta \Rightarrow \alpha^{-1} \cdot \alpha<\zeta \cdot \alpha .
$$

Hence, the product $\zeta \cdot \alpha$ is greater than 1 . In the same manner, we can show that the product $\zeta \cdot \alpha^{2}$ is an infinite surreal number, as it is greater than $\alpha$.

Acknowledgements We are grateful to John T. Baldwin for helpful comments on earlier version of the article. 


\section{References}

[1] P. Błaszczyk, Nota o rozprawie Otto Höldera "Die Axiome der Quantität und die Lehre vom Mass", Annales Universitatis Paedagogicae Cracoviensis. Studia ad Didacticam Mathematicae Pertinentia, vol. 5 (2013), pp. 129-144.

[2] P. Blaszczyk, A purely algebraic proof of the fundamental theorem of algebra, Annales Universitatis Paedagogicae Cracoviensis. Studia ad Didacticam Mathematicae Pertinentia, vol. 8 (2016), pp. 5-21.

[3] P. Błaszczyk, J. Major, Calculus without the concept of limit, Annales Universitatis Paedagogicae Cracoviensis. Studia ad Didacticam Mathematicae Pertinentia, vol. 4, (2014) pp. 19-38.

[4] P. Błaszczyk, K. Mrówka, Euklides i Arystoteles o ciqgtości. Część I. Euklides, Filozofia Nauki, vol. 21 (2013), pp. 91-115.

[5] B. Bolzano, Rein analytischer Beweis des Lehrsatzes, daß zwischen je zwei Werthen, die ein entgegengesetzes Resultat gewähren, wenigstens eine reelle Wurzel der Gleichung Liege, Gottlieb Hasse, Prague (1817).

[6] B. Bolzano, Paradoxien des Undendlichen, Reclam, Leipzig (1851).

[7] B. Bolzano, Theorie der reellen Zahlen in Bolzanos handschriftlichen Nachlasse, Hrsg. K. Rychlik, Tschechoslovakien Akademie der Wissenschaften, Prague (1962).

[8] B. Bolzano, Reine Zahlenlehre, [in:] J. Berg (Hrsg.), Bernard Bolzano Gesamtausgabe, Bd. 2A8, Frommann-Holzboog, Stuttgart (1976).

[9] K. Borsuk, W. Szmielew, Foundations of Geometry, NHPC, Amsterdam (1960).

[10] N. Bourbaki, Théorie de la mesure et de l'intégration. Introduction, Université Henri Poincaré, Nancy (1947).

[11] G. Cantor, Gesammelte Abhandlungen mathematischen und philosophischen Inhalts, Springer, Berlin (1932).

[12] G. Cantor, Über die einfachen Zahlensysteme, Zeitschrift für Mathematik und Physik, Neunzehnter Jahrgang, vol. 14 (1869), pp. 121-128, [in:] [11, pp. 35-42].

[13] G. Cantor, Zwei Sätze über eine gewisse Zerlegung der Zahlen in unendliche Produkte, Zeitschrift für Mathematik und Physik, Neunzehnter Jahrgang, vol. 14 (1869), pp. 152-158, [in:] [11, pp. 43-50].

[14] G. Cantor, Über die Ausdehnung eines Satzes aus der Theorie der trigonometrischen Reihen (1872), [in:] [11, pp. 92-102]. 
[15] G. Cantor, Grundlagen einer allgemeinen Mannigfaltigkeitslehre (1883), [in:] [11, pp. 165-208].

[16] G. Cantor, Mitteilungen zur Lehre von Transfiniten (1887), [in:] [11, pp. 378-439].

[17] G. Cantor, Beiträge zur Begründung der transfiniten Mengenlehre (1895), [in:] [11, pp. 285-311].

[18] G. Cantor, Beiträge zur Begründung der transfiniten Mengenlehre (1897), [in:] [11, pp. 312-351].

[19] L. C. Cohen, G. Ehrlich, The Structure of the Real Number System, Van Nostrand, Toronto (1963).

[20] J. H. Conway, On numbers and games, Academic Press, London (1976).

[21] J. H. Conway, On Numbers and Games, AK Peters, Natick (2001).

[22] J. W. Dauben, Georg Cantor. His Mathematics and Philosophy of Infinite, Princeton University Press, Princeton (1990).

[23] R. Dedekind, Stetigkeit und irrationale Zahlen, Friedrich Vieweg \& Sohn, Braunschweig (1872).

[24] R. Descartes, La Géométrie, Leiden (1637).

[25] P. Du Bois-Reymond, Die allgemeine Functiontheorie, Lauppschen, Tübingen (1882).

[26] P. Du Bois-Reymond, Théorie Générale des Fonctions, Nice (1887).

[27] P. Ehrlich, The Rise of non-Archimedean Mathematics and the Roots of a Misconception I: The Emergence of non-Archimedean Systems of Magnitudes, Archive for the History of Exact Science, vol. 60 (2006), pp. 1-121.

[28] P. Ehrlich, The Absolute Arithmetic Continuum and the Unification of all Numbers Great and Small, Bulletin of Symbolic Logic, vol. 18(1) (2012), pp. $1-45$.

[29] L. Euler, Introductio in Analysin Infinitorum, Lausanae (1748).

[30] L. Euler, Institutiones Calculi Differentialis, Saint Petersburg (1755).

[31] W. Ewald, From Kant to Hilbert, vol. II, Clarendon Press, Oxford (1996).

[32] H. Gonshor, An Introduction to the Theory of Surreal Numbers, Cambridge University Press, Cambridge (1986).

[33] H. Grassmann, Lehrbuch der Arithmetik, Enslin, Berlin (1861).

[34] R. Harshorne, Geometry: Euclid and Beyond, Spinger, New York (2000).

[35] H. Hankel, Zur Geschichte der Mathematik in Altertum und Mittelalter, Teubner, Leipzig (1874). 
[36] T. L. Heath, The works of Archimedes, Dover, New York (1912).

[37] J. L. Heiberg, Archimedis Opera Omnia cum Commentariis Eutocii, vol. I-II, Teubner, Leipzig (1880-1881).

[38] J. L. Heiberg, Euclidis Elementa, Teubneri, Lipsiae (1883-1888).

[39] G. Hessenberg, Grunbegriffe der Mengenlehre, Vandenhoeck und Ruprecht, Göttingen (1906).

[40] D. Hilbert, Grundlagen der Geometrie. Festschrift zur Feier der Enthüllung des Gauss-Weber Denkmals in Göttingen, Teubner, Leipzig (1899).

[41] D. Hilbert, Über den Zahlbegriff, Jahresbericht der Deutschen Mathematiker-Vereinigung, vol. 8 (1900), pp. 180-184.

[42] D. Hilbert, S. Cohn-Vossen, Anschauliche Geometrie, Springer, Berlin (1932).

[43] O. Hölder, Die Axiome der Quantität und die Lehre vom Mass, Berichte über die Verhandlungen der Königlich Sächsischen Gesellschaft der Wissenschaften zu Leipzig. Mathematisch-Physische Classe, vol. 53 (1901), pp. 1-63.

[44] O. Hölder, The Axioms of Quantity and the Theory of Measurement, Translated by J. Mitchell, Journal of Mathematical Psychology, vol. 40 (1996), pp. 235-252.

[45] H. Meschkowski, W. Nilson Hrsg., Georg Cantor - Briefe, Springer, Berlin (1991).

[46] K. Shamseddine, M. Berz, Analysis on the Levi-Civita field, a brief overview, [in:] Contemporary Mathematics 508, American Mathematical Society (2010), pp. 215-237.

[47] O. Stolz, Vorlesungen über Allgemeine Arithmetik, Teubner, Leipzig (1885).

[48] O. Stolz, J. A. Gmeiner, Theoretische Arithmetik, Teubner, Leipzig (1902).

[49] H. Weber, Lehrbuch der Algebra, Vieweg, Braunschweig (1895).

Pedagogical University of Cracow

Institute of Mathematics

Podchorążych 2

30-084 Cracow, Poland

e-mail: \{piotr.blaszczyk, marlena.fila\}@up.krakow.pl 